\title{
Irreducible Poster lateral Knee Dislocation: A Diagnostic Challenge.
}

\author{
${ }^{1}$ Prashant Acharya, ${ }^{2}$ Jagadish Surannavar \\ 1,2202, West End Manor, Kankanady Bypass road, Kankanady, Mangalore-575002 Karnataka
}

\begin{abstract}
Irreducible posterolateral knee dislocations are extremely rare and pose a significant diagnostic challenge to orthopaedic surgeons. Lack of awareness of this entity and its presentation, commonly result in a missed diagnosis and delayed treatment. We present a case of 40 year old man who had a low energy trauma to his knee and was initially diagnosed and treated as a case of patellar dislocation. Only after a thorough review of mechanism of injury, clinic-radiological findings and literature we could diagnose and treat this injury correctly.
\end{abstract}

Key words: Irreducible knee dislocation, Posterolateral, Dimple sign, Low energy trauma.

\section{Introduction}

More than $50 \%$ of acute knee dislocations may reduce spontaneously in the field ${ }^{1}$ and most of the remaining can be reduced by closed methods. Irreducible posterolateral knee dislocations form a very small percentage of knee dislocations. ${ }^{2,4,9,10,13,14}$ Although some pathognomic clinical signs have been described, the clinic-radiological features may not be very dramatic. ${ }^{3,4}$ If this specific entity is not kept in mind, it is difficult to diagnose posterolateral knee dislocations, given the rarity of this injury.

\section{Case report}

A 40 year old man was brought to the casualty of our institute with severe pain and deformity of his left knee. While trying to ward off a falling branch of a tree he sustained a twisting injury to the knee. On clinical examination the knee was in 30-40 degree flexion and a laterally subluxed patella seemed to be the only dramatic finding. Radiological evaluation also confirmed a laterally subluxed patella (Fig.1). The patella was reduced in the casualty without anaesthesia by simply extending the flexed knee and a plaster slab was applied. However check x-rays revealed a persistently subluxed patella. So a thorough review of history, clinicradiological findings and literature was done. The injury was sustained with the knee flexed in valgus and external rotation of leg. Re-examination of the patient revealed a transverse furrow at the medial joint line (Fig.3) and a lack of terminal extension. MRI showed complete disruption of both the cruciate ligaments and all the medial structures including MCL (Fig.2). With this information a diagnosis of irreducible posterolateral dislocation of knee associated with patellar subluxation was made. No neurovascular deficit was present and since it was a low velocity injury a decision was made to monitor the patient clinically for subsequent vascular compromise.

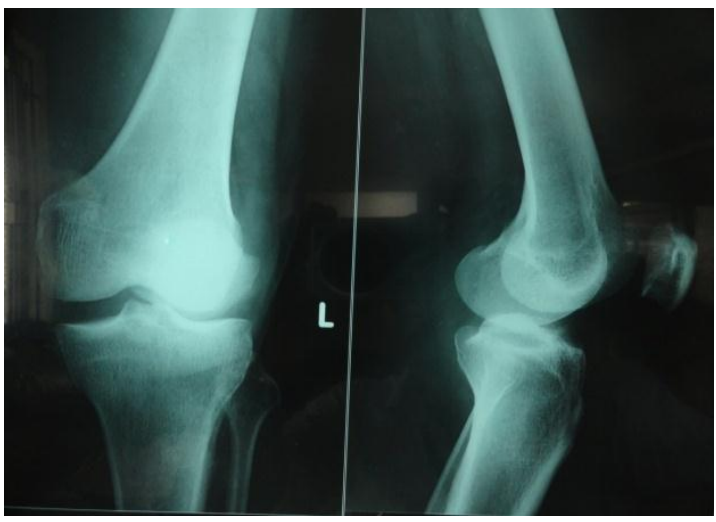

Fig.1

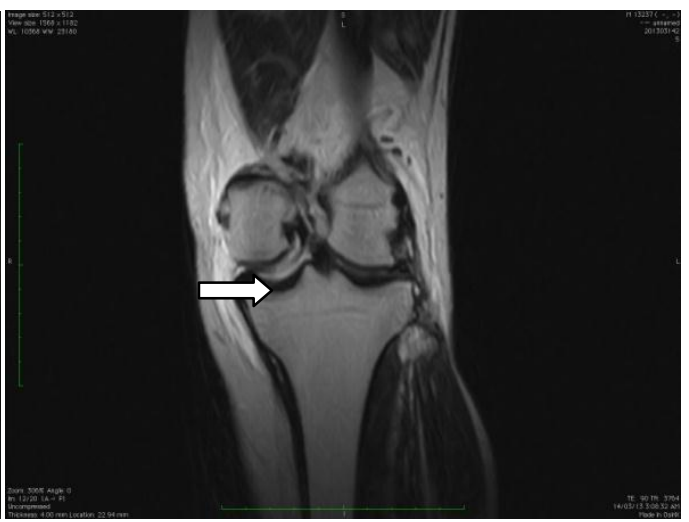

Fig.2

Patient was taken to the operating room and a closed reduction was attempted under general anaesthesia. However reduction could not be achieved due to elastic fixation. We decided to proceed with open reduction through a standard midline longitudinal incision. The medial femoral condyle was located subcutaneously, having button-holed through an ' $\mathrm{L}$ ' shaped tear in the medial retinaculum. The superior margin 
of the lower flap had invaginated into the joint (Fig.4). Reduction could be achieved only after extraction of the invaginated soft tissue. Both the cruciates were found to be torn with gross antero-posterior and medial instability. The posterolateral structures were intact.

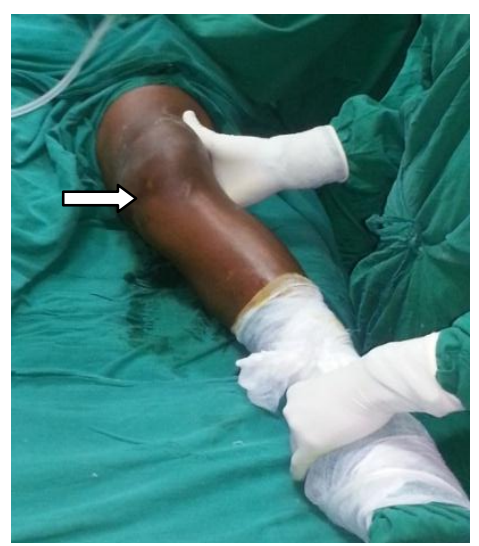

Fig.3

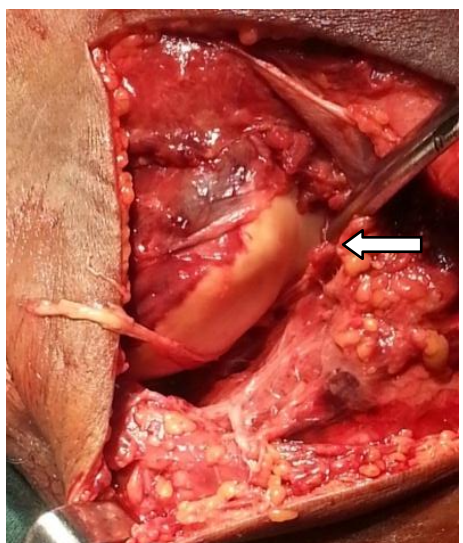

Fig.4

A primary repair of all the medial structures was done and the limb was splinted with a knee brace in 15 degree flexion. The neurovascular status remained same post-operatively (Fig.5). Gradually range of movement exercises were started. The patient had regained 90 degree of flexion at one month post-operative follow-up and there was grade 3 antero-posterior and grade 1 medial laxity. At this stage arthroscopic ACL and PCL reconstruction was done using BPTB graft for ACL and hamstring tendon graft for PCL (Fig.6). Evaluation at the end of the procedure revealed adequate medial stability and no further medial reconstruction was done. Further rehabilitation proceeded as per the standard protocol of our department.

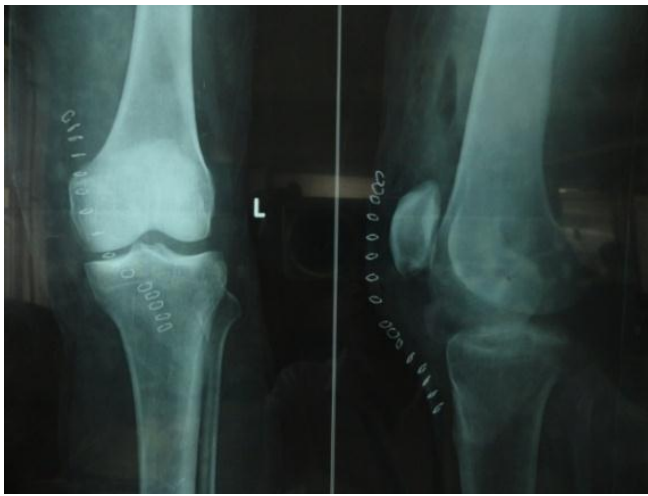

Fig.5

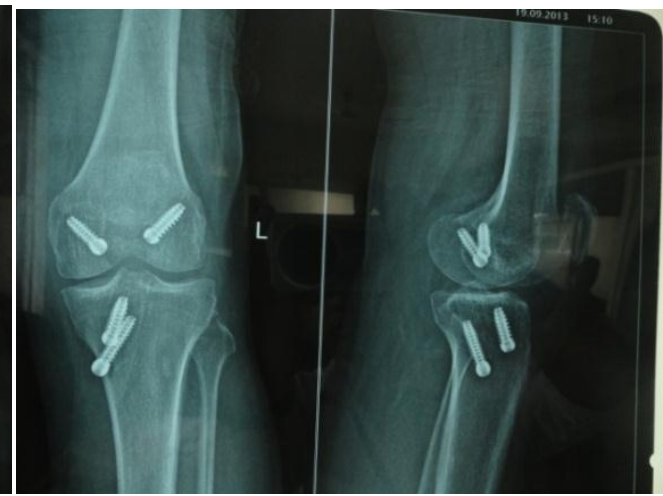

Fig.6

Patient was followed up at regular intervals and at 6 months he had a pain free range of movement of 0 to 120 degree with normal patellar tracking and no extensor lag. There was no antero-posterior instability with grade 1 medial laxity.

\section{Discussion:}

Knee dislocations are rare but devastating injuries due to the involvement of multiple structures and potentially serious complications. Based on the mechanism of injury they have been classified as high and low injuries, with a new type, ultra-low velocity dislocations being recently added. ${ }^{5,6,7}$ Knee dislocations are also classified based on displacement of tibia in relation to the femur. ${ }^{8}$ Rotary dislocations form a very small percentage of knee dislocations, but account for most of the irreducible dislocations. ${ }^{9}$ Posterolateral dislocations are the most common rotary dislocations. ${ }^{10}$

Posterolateral knee dislocation is caused by application of a valgus force to a flexed knee with external rotation of the tibia. ${ }^{3,11}$ The medial femoral condyle button-holes through the medial capsule as a flexed knee is forced into severe valgus. As the deforming force is dissipated, the knee partially reduces. And in doing so, the medial femoral condyle pushes the torn medial structures into the joint resulting in the classical finding of invaginated medial structure. The external rotation of the tibia with respect to the femur is associated with lateral displacement of the patella as occurred in the case presented here. ${ }^{3,12}$

Although rare, irreducible posterolateral knee dislocations present with certain well defined features. The puckering of the skin at the medial joint line has been called the "Dimple sign". ${ }^{13}$ In a co-operative patient it 
may be possible to palpate for the subcutaneously located medial femoral condyle. Lateral patellar subluxation or dislocation and lack of terminal extension may also be present. Since these are low-energy injuries the risk of neurovascular complications is less. ${ }^{3,4,13}$ However the patient should be properly evaluated for neurovascular compromise at presentation and should be monitored by non invasive methods peri-operatively. ${ }^{11}$

Radiographs may not show any dramatic displacement, however a slight widening of the medial tibiofemoral joint may be present on the AP view due to soft tissue interposition. ${ }^{3}$ AP view may also show some lateral displacement of the tibia in relation to the femur. ${ }^{4}$ The lateral view may show slight posterior subluxation of the medial tibial plateau. ${ }^{4}$ MRI reveals the soft tissue interposition into the medial tibio-femoral compartment and inter-condylar notch apart from the ligament injuries. ${ }^{14}$ MR Dimple sign has also been described to avoid misinterpretation of the invaginated medial capsule and MCL as the torn meniscus. ${ }^{15}$

Dislocations require prompt diagnosis and management to avoid disabling complications. Once a diagnosis of irreducible posterolateral knee dislocation has been made treatment should be directed at emergent reduction of the joint. Attempts at closed reduction are usually met with failure due to elastic fixation and repeated attempts may increase the risk of necrosis of skin overlying the medial femoral condyle. ${ }^{10,13}$ Prompt open reduction, which can be easily achieved by sectioning the invaginated soft tissue, should be the first line of management. Reconstruction of the torn ligaments can be done primarily or as delayed reconstruction based on treating surgeon's choice. ${ }^{6,9,16}$

\section{Conclusion:}

Although an increasing number of case reports are being published on irreducible posterolateral knee dislocations, these injuries still remain a novelty for majority of orthopaedic surgeons. We would like to emphasize on eliciting an exact mechanism of injury in every case of knee trauma as this might raise a suspicion towards the possibility of these rare injuries. Since there is a lack of dramatic displacement clinically as well as radiologically, it is important to look for the Dimple sign, the subcutaneously located medial femoral condyle, the loss of terminal extension and sometimes the associated laterally displaced patella. We believe that the risk of vascular injury in these dislocations is less and clinical monitoring of the vascular status may be adequate. This may be supplemented by non-invasive methods pre and post-operatively if an immediate ligament reconstruction is planned. Initial treatment is always open reduction and further ligament reconstruction protocol remains individual surgeon's choice.

\section{References}

[1]. Montgomery JB. Dislocation of the knee. Orthop Clin North Am 1987; 18: 149

[2]. Griswold AS. Irreducible dislocations of the knee joint. J Bone Joint Surg Am 1951; 33: 787-791.

[3]. Huang FC, Simonian PT, Chansky HA. Irreducible posterolateral dislocation of the knee. Arthroscopy 2000; 16: 323-327.

[4]. Quinlan AG, Sharrad WJW. Posterolateral dislocation of the knee with capsular interposition. J Bone Joint Surg Br 1958; 40: 660663.

[5]. Green NE, Allen BL. Vascular injuries associated with dislocation of the knee. J Bone Joint Surg Am 1977; 59: 236-239.

[6]. Shelbourne KD, Porter DA, Clingman JA, McCarrol JR, Rettig AC. Low velocity knee dislocation. Orthop Rev 1991; 20: 9951004.

[7]. Azar FM, Brandt JC, Miller RH, Phillips BB. Am J Sports Med 2011; 39: 2170-2174.

[8]. Kennedy JC. Complete dislocation of the knee. J Bone Joint Surg Am 1963; 45: 889.

[9]. Shields L, Mital M, Cave EF. Complete dislocation of the knee: Experience at the Massachusetts general hospital. J Trauma1969; 9: 192-215.

[10]. Hill JA, Rana NA. Complications of posterolateral knee dislocations: Case report and review of the literature. Clin Orthop 1981; 154: 212.

[11]. Urguden M, Bilbasar H, Ozenci AM, Akyildiz FF, Gur S. Irreducible posterolateral knee dislocation resulting from a low energy trauma. Arthroscopy 2004; 20: 50-53.

[12]. Van Thiel GS, Baker CL, Joseph CB. A chronic posterolateral knee and patella dislocation: Case report. J Orthop Trauma 2009; 23: 541-545.

[13]. Wand JS. A physical sign denoting irreducibility of a dislocated knee. J Bone Joint Surg Br 1989; $71: 862$.

[14]. Tsiagadigui JG, Sabri F, Sintzoff S, Schuind F. Magnetic resonance imaging for irreducible posterolateral knee dislocation. J Orthop Trauma 1997; 11: 457-460.

[15]. Harb A, Lincoln D, Michaelson J. The MR dimple sign in irreducible posterolateral knee dislocations. Skeletal Radiology 2009; 38: 1111-1114.

[16]. Sisto DJ, Warren RF. Complete knee dislocation: A follow-up study of operative treatment. Clin Orthop 1985; 198: 94-101.

Legends to figures:

Figure 1: The anteroposterior and lateral radiographs of the injured left knee showing lateral patellar subluxation with no other dramatic finding.

Figure 2: MRI image showing soft tissue interposition into the medial compartment of the knee joint.

Figure 3: Dimple sign: Transverse furrow at the medial joint line.

Figure 4: Invaginated medial soft tissue structures.

Figure 5: Postoperative radiograph after initial open reduction and medial repair.

Figure 6: Postoperative radiograph after delayed ligament reconstruction. 